\title{
OMICIDI, INGIURIE, CONTENZIOSI: VIOLENZA VERBALE E FISICA NELLA CALABRIA DEL XV SECOLO
}

\author{
MURDERS, INJURIES, ALTERCATIONS: \\ VERBAL AND CORPORAL VIOLENCE \\ IN THE 15th CENTURY CALABRIA (ITALY)
}

\section{Gemma TeResa Colesanti \\ IBAM-CNR \\ DANIELA SANTORO Università di Palermo ${ }^{1}$}

\begin{abstract}
Riassunto: Il ritrovamento, presso l'Archivio di Stato di Napoli, dell'unico registro superstite della serie Justitiae della Cancelleria aragonese (1453-1454), ha permesso attraverso l'analisi delle denunce - reati contro l'amministrazione, contro la persona, reati di natura sessuale e contro lo stato- di ricostruire uno spaccato della società calabrese.
\end{abstract}

Parole chiave: Giustizia; Denunce; Calabria; Donne; Violenza, Ingiurie.

\begin{abstract}
The finding, in the "Archivio di Stato" of Napoli, of the only surviving Justitiae register belonging to the "Cancelleria aragonese" serie "s (1453-1454), led to insight into the Calabria society thruough the analysis of the denunciations of crimes, such as: crimes against government, administration or people and sexuals crimes.
\end{abstract}

Key words: Justice; Denunciations; Calabria, Women; Violence, Injuries.

\section{SOMMARIO}

1. Alfonso il Magnanimo e la giustizia nel Regno: il 'caso Calabria'.- 2. Violenza delle donne, violenza contro le donne.- 3 . Vita in Calabria nel XV secolo: uno spaccato sociale.

L'Archivio di Stato di Napoli, nel fondo "Camera della Sommaria", tra i conti erariali -introiti ed esiti che in qualità di esattori del Fisco, gli erari presentavano per i proventi dei feudi devoluti- conserva, unico superstite, un registro della serie Justitiae della Cancelleria aragonese: Quaternus denunciacionum anni II indicionis MCCCCLIIII Curie domini

\footnotetext{
${ }^{1}$ Gemma Teresa Colesanti è autrice dell'introduzione e del $2^{\circ}$ paragrafo. Daniela Santoro è
} autrice del $1^{\circ}$ e $3^{\circ}$ paragrafo. 
viceregis Calabrie $^{2}$. Il registro, in una minuscola corsiva di tipo notarile, contiene 307 denunce, per un totale di 90 carte con numerazione progressiva moderna, senza recto e verso: le parti che si riferiscono al formulario giuridico sono in latino, in volgare quelle che riportano le testimonianze dirette, in un latino volgarizzato sono parecchie parti che traducono e raccontano i motivi e le occasioni delle accuse.

Relative tutte al territorio calabro, le denunce si estendono lungo un arco temporale che va dal 12 settembre 1453 al 28 agosto 1454 e abbracciano una vasta gamma di reati:

- reati contro la persona: aggressioni, sequestri e tentativi di omicidio; ingiurie verbali (spergiuri, bestemmie), icasticamente definite da Nada Patrone «i graffiti della mentalità ${ }^{3}$.

-reati di natura sessuale: adulteri, lenocinio, maltrattamenti, stupro e violenza sessuale;

- reati contro lo stato: lesa maestà, fabbricazione di monete false;

-reati contro il patrimonio: mancato rispetto dei termini pattuiti nei prestiti (si tratti di bestie o di denaro); appropriazioni indebite e sconfinamenti; falsificazioni di ricevute; furti (di olive, legna, grano; di animali di piccola taglia: galline, porcellini; e grande: buoi); gioco d'azzardo.

Scarni al punto da rendere scivoloso il tentativo di seguire in dettaglio l'andamento o gli esiti delle vicende giudiziarie, i dati che trapelano dalle denunce riescono a essere eloquenti, letteralmente: la forza delle parole in volgare di accusati e accusatori offre la possibilità di soffermarci su vicende parentali e personali, su identità reali o attribuite, su concrete e dense situazioni di vita; una fonte che permette, per la sua stessa tipologia, una ricostruzione dalle molteplici dimensioni, dalla storia del costume e della società a quella del diritto e dell'economia. Non solo: consente di visualizzare in modo netto e preciso intere fette di territorio calabrese dallo Ionio al Tirreno, e aggiunge piccoli tasselli alla conoscenza topografica della zona ${ }^{4}$; al contempo, gli indubbi rapporti tra storia del diritto e storia sociale, consentono una messa a fuoco di aspetti legati a temi specifici: gli ebrei di

${ }^{2}$ Archivio di Stato di Napoli, Dipendenza della Sommaria, Quaternus, vol. 631/2 (d'ora in poi, ASN, Dipendenza, vol. 631). Il registro fu segnalato da Ernesto PONTIERI, La Calabria a metà del secolo XV e le rivolte di Antonio Centelles, Napoli, 1963, p. 45. Sulla Regia Camera della Sommaria, massima magistratura finanziaria del Regno di Napoli, organo generale di controllo di tutto l'impianto giudiziario, economico e amministrativo della monarchia, e sulle sue origini, v. Roberto DELLE DONNE, Alle origini della Regia Camera della Sommaria, in «Rassegna Storica Salernitana», 15 (1991), pp. 25-61.

${ }^{3}$ Anna Maria NADA PATRONe, Simbologia e realtà nelle violenze verbali del tardo Medioevo, in «Simbolo e realtà della vita urbana nel tardo Medioevo», a c. di Massimo MIGLIO e Giuseppe LOMBARDI, Roma, 1993, p. 50.

${ }^{4} \mathrm{Su}$ storia ed economia calabrese in età aragonese cfr. Salvatore FODALE, La Calabria angioino-aragonese, in "Storia della Calabria medievale. I quadri generali», a c. di Augusto PLACANICA, Roma, 2001, pp. 183-262; Alfonso LEONE, I mercanti forestieri in Calabria e la struttura economica della regione, loc. cit. . pp. 523-534. Sul peso dell'eredità aragonese ai fini di uno sviluppo successivo, Giuseppe ĠALASSO, Economia e società nella Calabria del Cinquecento, Napoli, 1992, pp. 87 ss. 
Calabria per esempio ${ }^{5}$, o i rapporti affaristici e matrimoniali con aree geografiche vicine, tra tutte l'Albania. Uno studio agli inizi e che in questa sede viene presentato in una veste di 'cantiere aperto', con grappoli di problemi da affrontare e chiarire, parallelismi e confronti da verificare con altre aree geografiche, mediterranee e non ${ }^{6}$.

Sull'amministrazione e organizzazione della giustizia nel Regno in particolare durante l'età alfonsina, abbiamo constatato una carenza di studi, dovuta anche all'esiguità di serie documentarie complete, a differenza di quanto negli ultimi anni si è prodotto per l'Italia centro-settentrionale ${ }^{7}$.

La lettura delle carte del nostro registro riserva la possibilità di un'immersione nella vita di una parte del Regno, la Calabria, gravata nel corso dei secoli da calamità naturali, carestie, violenze sotterranee e dichiarate, rivolte baronali. Terra in cui regnano, sin dai primi decenni del XV secolo, miseria «morale e materiale»e anarchia, «nella vita pubblica e privata»; terra che come «ogni altra regione del regno di Napoli, e più delle altre per le lotte accanite» combattute sul suo suolo, sente forte il «bisogno di assestamento e di pace» ${ }^{8}$, specie nei difficili e disordinati anni in corrispondenza del regno di Giovanna II: a quel clima di conflittualità, dialettica e armata, Alfonso V -padrone di Napoli nel giugno 1442 - era convinto di porre rimedio.

\footnotetext{
${ }^{5}$ Sugli ebrei in Calabria, Cesare Colafemmina, Per la storia degli ebrei in Calabria: saggi e documenti, Soveria Mannelli, 1996; Sonia VIVACQUA, Calabria, in «L'ebraismo dell'Italia meridionale peninsulare dalle origini al 1541: società, economia, cultura», a c. di Cosimo Damiano FoNSECA, Galatina, 1996, pp. 295-310; Gemma Teresa COLESANTI, Documenti per la storia degli ebrei in Calabria nel secolo XV, in "Hebraica hereditas. Studi in onore di Cesare Colafemmina», a c. di Giancarlo LACERENZA, Napoli, 2005, pp. 27-31.

${ }^{6} \mathrm{~L}$ 'edizione del registro è prevista nel XIV volume, serie II delle Fonti Aragonesi, pubblicate dall'Accademia Pontaniana.

${ }^{7}$ Per quanto riguarda le istituzioni dell'Italia centro-meridionale un classico rimane Gennaro Maria MónTI, Le origini della Gran Corte della Vicaria e le codificazioni dei suoi riti. Bari 1929; v. inoltre Giovanni CASSANDRO, Il comune meridionale nell"Etä Aragonese in "Ätti del Congresso Internazionale di Studi sull'Età Aragonese», Bari, s.a.; Giovanni CASSANDRO Lineamenti del diritto pubblico del Regno di Sicilia «citra Farum» sotto gli Aragonesi. Bari, 1934 Antonio ALLOCATI, Lineamenti delle istituzioni pubbliche nell'Italia meridionale. Parte prima. Dall'età prenormanna al viceregno spagnolo, Roma, 1968; Alan RYDER, El reino de Nápoles en la época de Alfonso el Magnánimo, Valencia, 1987, il capitolo 5 dedicato alla giustizia, pp.163199 . In particolare per la Sicilia, v. gli studi di Antonino GIUFFRIDA, La giustizia nel Medioevo siciliano, Palermo, 1975; Beatrice PASCIUTA, In regia curia civiliter convenire: giustizia e città nella Sicilia tardomedievale, Torino, 2003. Per i territori della Corona d'Aragona, segnaliamo Jesús LALINDE ABADIA, La jurisdicción real inferior en Cataluña: "corts, veguers, battles", Barcelona, 1966; Maria Dolors FARRENY I SISTAC, Processos de crims del segle XV a Lleida: Transcripció $i$ estudi lingüístic, Lleida, 1986; Prim BERTRAN I ROIGÉ, Els llibres del Battle de Cervera, Galzeran Sacirera (1459-1460). Notes de vida quotidiana i conflictivitat urbana a Cervera, a darreries de l'edat mitjana, in "Acta Historica et Archaeologica Mediaevalia», 26, Facultat de Geografia i Història Universitat de Barcelona, Barcelona, 2005, pp. 875-901. Per l'Italia settentrionale un punto di partenza è Andrea ZORZI, Giustizia criminale e criminalità nell'Italia del tardo medioevo: studi e prospettive di ricerca, in "Società e storia», a. XI, 46 (1989), pp. 923-965; da segnalare inoltre gli atti del Convegno svoltosi a Trento nel 1999: Criminalità e giustizia in Germania e in Italia. Pratiche giudiziarie e linguaggi giuridici tra tardo medioevo ed età moderna, Bologna, 2001; per il Veneto, Alfredo VIGIANO, Fonti e studi su istituzioni giudiziarie, giustizia e criminalità nel Veneto del Basso Medioevo, in «Ricerche Storiche», XX, I (1990), pp. 131-149.

${ }^{8}$ PONTIERI, La Calabria, pp. $12 \mathrm{~s}$.
} 


\section{Alfonso il Magnanimo e la GiUStizia NEL Regno: IL 'CASO CALABRIA'}

Re di Aragona, Sicilia e Napoli, Alfonso V il Magnanimo in alcuni tra i documenti superstiti conservati a Napoli, si pronunciava in merito al problema della giustizia: il dovere di un re verso i sudditi era in primo luogo, secondo l'ideale alfonsino, una buona amministrazione della giustizia. Particolarmente significativo un testo del 1449 in cui trapela la preoccupazione del re per la situazione vigente ma al contempo la ferma convinzione di stare operando nel giusto, unita alla certezza che i propri ideali di giustizia abbiano trovato pratica e applicazione, tanto da avere sconfitto annosi e complessi problemi del Regno, ad esempio il brigantaggio:

Cum post acquisitionem huius praetiosissimi viridarii nostri regni Sicilie citra farum tot laboribus periculis et expensis, Deo actore, ac Sanctissima Ecclesia Romana approbante, iustissimo titulo a manibus tyrannorum et plurium invasorum recuperavimus et omnes guerrarum calamitates pacis lenitate sopivimus incredibilem itineribus et portubus securitatem praestando, bonos meritis exaltando, facinorosque et execrabiles ultore gladio e vita exterminando, omnes cogitationes et studia quamplures noctes vigilia transeuntes interposuimos ${ }^{9}$.

Agli ambasciatori catalani in visita nel 1450 , il re offriva una smagliante visione della giustizia e dell'ordine che regnavano sul suolo italiano:

Fa e ministre per si e sos officials e ministres axi continuament justicia que jatsia ans quel conquistas hi hagues moltes guerres e dissensions no solament dels stranys ab los regnicoles del dit Realme, mas encare entre los regnicoles dessus dits, e si perpetraven e cometien en los poblets e per camins homicidis furts roberies, e molts altres crims, e justicia noy era administrada, empero la sua Maesta apres la dita conquista no solament ha donat pau e extirpacio de tota guerra entre los regnicoles mas encare entre los regnicoles e los stranys e axi extirpats tots crims maleficis e delictes que les gents axi del dit Realme com strangers ab llurs diners e robes van publicament per lo dit Realme e per los camins e boschs de aquell de nit e de dia segurament e quiete que no han redupte o temor de mal o sinistre algu, nols es feta injuria o molestia alguna ${ }^{10}$.

${ }^{9}$ Citato da RYDER, El reino, pp. $189 \mathrm{~s}$.

${ }^{10}$ Ibidem, p. $190 \mathrm{~s}$. Supremo tribunale del Regno è il Sacro Regio Consiglio, composto da un numero variabile di consıglieri e assistenti, più un presidente. Ogni consigliere dẹcide le cause, portate davanti al re, in nome di questi. Al Sacro Consiglio si ricorre da qualsiasi tribunale del Regno, e dietro supplica, il re a volte avoca a sé e al suo Consiglio cause pendenti davanti ad altri giudici. A volte l'istruzione è commessa al capitano del luogo dove è sorta la questione; il re rende poi esecutiva la sentenza. In periferia la competenza penale di primo grado è del capitano tra le nostre carte ad esempio, compare il capitano di Policastro e Rocca di Neto, ASN, Dipendenza della Sommaria, Quaternus, c. 8 ) che si trova in ogni citta demaniale, ma le sẹntenze di una certa importanza non divengono esecutive senza riesame da parte dell'udienza provinciale. Quanto alla competenza civile è del capitano e dei giudici cittadini e in piccola parte dei baiuli, AllOCATI, Lineamenti, pp. $72 \mathrm{~s}$. 
Uno scenario ideale, destinato a uno scarto inevitabile rispetto a quello reale. Il brigantaggio che Alfonso combatteva nella convinzione di essere prossimo a centrare l'obiettivo di sradicarlo, rimaneva una delle piaghe della Calabria quattrocentesca: qui regnava «la delinquenza, che infieriva, sia come fatto individuale, sia nella forma sociologicamente più grave dell'associazione di malviventi a scopo prevalentemente di estorsione ${ }^{11}$. Crotone, Cosenza, Amantea, Montalto: centri importanti le cui strade di accesso, traspare dalle carte del nostro registro, sono poco sicure, con conseguenze dannose per i commerci; nel maggio 1454 Stefano, figlio di Angelo Mansa de Fillino, veniva denunciato da un servitore della Curia, Guglielmo Gardo che era stato assalito lungo la strada che portava a Mangone, a sud di Cosenza: estratta prima la curtellissa che Guglielmo teneva a fianco e tentato con questa di percuotere lo stesso Guglielmo, l'accusato lo colpiva cum pugillo in facie e gli scagliava contro quamplures lapides ${ }^{12}$. Mentre passeggiava pacifice et quiete lungo il fiume in prossimità di Borrello, vicino Reggio Calabria, nel gennaio 1454 un ebreo di Monteleone, Silagayo, veniva colpito da Masi di Lentini cum quadam macza e ferito in diversis partibus corporis ${ }^{13}$. Riusciva a scampare all'agguato Tommaso Matera di Cosenza, assalito da cinque uomini armati armis prohibitis mentre passava per viam puplicam in prossimità di Tarsia ${ }^{14}$.

Non sono rari i casi di sequestro. Tenuto cum compedibus ferreis in pedibus Pietro Labruto denunciava Nardo Bonofacio di Crotone il quale, animo malivolo, lo aveva fatto catturare e tenuto in captivitate otto giorni ad suum libidum voluntatis ${ }^{15}$. Antonio Rosellino di Paternò denunciava tre uomini di Crotone che armati baculis seu bastonis, e uno di loro cum barbussa ferrea, lo avevano aggredito mentre si trovava in un terreno in prossimità di Crotone a custodire del bestiame e lo avevano picchiato a sangue; legategli le mani cum quadam cordula, lo avevano condotto ad casalem Cutri e tenuto prigioniero alcuni giorni; lì avevano portato pure parecchi degli animali di cui Antonio era a guardia, tra cui due vacche gravide che i tre crotonesi avevano frustato tanto da farle abortire ${ }^{16}$.

Ad abusi e vessazioni ricorrono anche gli esponenti delle classi sociali più alte, baroni e marchesi che si macchiano di delitti e soprusi nei confronti dei propri uomini, un «brigantaggio signorile», secondo la definizione di Pontieri; era il caso di Tommaso Caracciolo, marchese di Gerace e conte di Terranova ${ }^{17}$, denunciato l'8 agosto 1454 da Giovanni Calamita per non avere

\footnotetext{
${ }^{11}$ PONTIERI, La Calabria, pp. $45 \mathrm{~s}$.

${ }^{12}$ ASN, Dipendenza, vol. 631, c. 57.

${ }^{13}$ ASN, Dipendenza, vol. 631, c. 36.

${ }^{14}$ ASN, Dipendenza, vol. 631 , c. 47.

${ }^{15}$ ASN, Dipendenza, vol. 631, c. 24.

${ }^{16}$ ASN, Dipendenza, vol. 631 , c. 34.

${ }^{17} \mathrm{Su}$ Caracciolo v. PONTIERI, La Calabria, pp. 49 ss., 57.
} 
rispettato un mandato del viceré ${ }^{18}$ : il marchese il 14 del mese, in base a quanto disposto dalla corte napoletana, veniva raggiunto da un ordine di arresto ${ }^{19}$.

Di una violenza efferata ad opera di uomini e donne appartenenti a tutti i gruppi sociali, il nostro registro offre un vasto panorama. Gli assalitori, animo irato et vultu tinto, cum animo irato et forabundus, si abbandonano a ogni tipo di offesa, preda di una furia inconsulta: mentre si trovava intus claustrum di San Francesco, frate Antonio Corraducio di Amantea veniva aggredito da Giovanni Lauro che, senza rispetto per la sacralità del luogo, lo afferrava per il petto e lo colpiva dicendo più volte in volgare: chi si tu et de chi si figlo, e ulteriore ingiuria, arribaldo gagloffo ${ }^{20}$. Nel corso di una rissa generale che aveva come scenario la piazza di Amantea, Antonio Cocza veniva denunciato da Roberto Cinnaro ripetutamente aggredito con zappa, bastone, pugnali: motus ad iram lo aveva preso per i capelli prostando ipsum ad terram et de eo faciendo id quod voluerit. Lo stesso Antonio veniva denunciato da Guglielmo Grimaldo che trovandosi a passare, e assistendo alla scena, bono zelo lo aveva redarguito: o Antoni Cocza non fai bene chi levi lo bastune ad quillo et dailo allo altro; parole che avevano scatenato la reazione di Antonio che animo malivolo et tinto vulto gli si era scagliato contro e gli aveva dato uno schiaffo. Denunciato, ancora, da Domenico Valle che venendo da fora e portandose una lanza sive bastone et portandolo in collo cum uno panaro arreto era stato assalito da Antonio che gli si era letteralmente lanciato sopra per levargli il bastone, dicendogli lassa quisso bastone si non chi avirai lo malo iorno, con l'aggiunta di due pugni alle spalle ${ }^{21}$.

Lo sfondo di tante denunce è costituito da un'alterazione dell'animo, quasi possessione estranea - spiritu diabolico instigatus - che si traduce in gesti e parole brutali e taglienti. "Questa rudezza di costumi, - scrive Pontieri- questa ferocia di fatti che si ritrovano nei più miseri strati della popolazione calabrese del Quattrocento, nella coscienza dei quali non arriva nessuna luce di spiritualità né dalla religione né dalla cultura, era ben nota fuori della regione e creava a questa una fama non lusinghevole $»^{22}$.

Terra, la Calabria, nei confronti della quale Alfonso il Magnanimo avrebbe nutrito sentimenti di risentimento e fastidio, spina nel fianco nel suo ben oleato congegno di amministrazione della giustizia. Emblematico -così riferisce il suo segretario, il Panormita- quanto era solito ripetere: volentieri avrebbe rinunciato al mestiere di re e preferito una vita da uomo comune piuttosto che avere a che fare con i calabresi che «nihil hominis habent praeter figuram ${ }^{23}$. L'azione di governo di Alfonso, in Calabria, era destinata a

\footnotetext{
${ }^{18}$ ASN, Dipendenza, vol. 631, c. 85.

${ }^{19}$ Sulle vicende successive all'ordine di arresto, PONTIERI, La Calabria, p. 52.

${ }^{20} \mathrm{ASN}$, Dipendenza, vol. 631, c. 7.

${ }^{21}$ ASN, Dipendenza, vol. 631, cc. 13-15.

${ }^{22}$ PONTIERI, La Calabria, p. 48.

${ }^{23}$ Antonius Panormita, De dictis et factis Alphonsi regis, Rostock, 1589, lib. I, p. 19.
} 
scontrarsi con incrostate e inveterate situazioni che l'avrebbero resa pressoché inefficace.

\section{VIOLENZA DELLE DONNE, VIOLENZA CONTRO LE DONNE}

Tra i possibili ambiti di approfondimento e studio che questa fonte calabrese offre, si è scelto in questa sede di concentrare l'attenzione sulla violenza in senso lato, con uno spazio ai casi che vedono per protagoniste, soggetti o oggetti, le donne ${ }^{24}$ : una casistica 'al femminile' che spazia dalle violenze fisiche a quelle verbali e in cui un ruolo è riservato anche alle testimoni, spettatrici dirette degli avvenimenti o informate sui fatti. La moglie di Nardo Magliari de Casali Yacti, Caterina, mentre si trovava in casa sub Dei pace et regia proteccione secura, era stata aggredita da Gaspare Murra -denunciato per questo da Nardo- che cum quadam arcute l'aveva colpita sul capo e altre parti del corpo con tale forza da ucciderla, se non fossero arrivati i soccorsi. Grazia, moglie di Antonio Crispino, mentre si trovava in casa veniva presa a pugni in faccia e in tutto il corpo da un tale di Rogliano che feritala e gettatala a terra le strappava i capelli ${ }^{25}$.

Vittime, le donne, di violenze fisiche ${ }^{26} \mathrm{ma}$, soprattutto oggetto di violenze verbali: attraverso le quali vengono fuori «l'atteggiamento psicologico, l'immaginario, la simbologia verbale, i valori ed i tabù dell'uomo medievale, i suoi moti di violenza più o meno repressa, le sue idiosincrasie» ${ }^{27}$. Suscitati, in maniera volontaria o no, dalle donne, gli insulti sono spesso rivolti più che a difetti fisici ad atteggiamenti morali. Se la figlia di Gregorio Falcazerio de casalis Czagarisii veniva accusata di avere lo diabolo in capo $^{28}$, la moglie di Tommaso Bonfiglio, mentre si trovava in plathea puplica, veniva

\footnotetext{
${ }^{24} \mathrm{Cfr}$. gli studi sull'argomento riguardanti aree diverse: Viviana RIzzO, Donne e criminalità a Viterbo nel XV secolo, in «Rivista storica del Lazio», 12 (2000) pp. 11-27; Alessandra PERI, "Ne delicta remaneant impunita": il caso di Aspra Sabina (oggi Casperia) nel tardo Medioevo, in «Rivista storica del Lazio», 18 (2003), pp. 39-56, in particolare pp. 47-50; Chiara CAMPESE, Violenza verbale, violenza femminile. Le donne nel tumulto contro l inquisizione a Bologna 1299, in "Nuova DWF, Quaderni di studi internazionali sulla donna" 16 (1981) pp. 119-133; Trasgressioni. Seduzione, concubinato, adulterio, bigamia (XIV-XVIII secolo), a c. di Silvana SEIDEL MENCHI e Diego QUAGLIONI, Bologna, 2004 ; Matrimoni in dubbio. Unioni controverse e nozze clandestine in Italia dal XIV al XVIII secolo, a c. di Silvana SEIDEL MENCHI e Diego OUAGLIONI, Bologna, 2001; Claude GAUVARD, De grace especial: crime, état et société en France a la fin du Moyen Age, Parigi 1991, pp. 299-346 ed in particolare le pp. 323-346.

${ }^{25} \mathrm{ASN}$, Dipendenza, vol. 631, cc. 5, 6.

${ }^{26}$ Una tipologia di reato, lo stupro, che tende a mantenere, ad esempio in Sicilia, le stesse caratteristiche nello spazio e nel tempo al punto da ipotizzare si tratti di accusa priva di fondamento, lanciata magari a scopi ricattatori GIUFFRIDA, La giustizia, p. 33. Sulle possibilità offerte dall'analisi del materiale giudiziario, ai fini di un'immagine del tutto reale della vita al femminile, Giovanna CASAGRANDE-Michela PAZZAGLIA, "Bona mulier in domo". Donne nel Giudiziario del Comune di Perugina nel Duecento, in «Annali della Facoltà di Lettere e Filosofia dell'Università degli Studi di Perugia. 2. Studi storico-antropologici», XXII (1998-1999), pp. 127166.

${ }^{27}$ NADA PATRONE, Simbologia e realtà, p. 50.

${ }^{28}$ ASN, Dipendenza, vol. 631, c. 18. Come le ingiurie, i soprannomi possono fissare difetti fisici e morali, vizi e tendenze, atteggiamenti e tratti caratteristici; v. Aniello GENTILE, Il soprannome nei documenti medioevali dell'Italia meridionale, in «Quaderni linguisici», 5 (1959), pp. 5-98.
} 
assalita verbalmente da Antonio de Blasio che ad eius iniuriam et infamiam et iacturam l'aveva minacciata: puctana ruffiana eo ti voglio taglare lo naso de la fache ${ }^{29}$. Minaccia che si era tradotta in realtà nel caso di Impernata, sorella di Matteo de Falico di Melissa cui quattro concittadini probabilmente imparentati, tutti di nome Pignatario, dopo averla colpita sul viso e sul capo pluribus ictibus mentre si trovava per strada, avevano troncato il naso ${ }^{30}$.

Una vicenda complessa, ricca di passaggi quella che, nel febbraio 1454, ha tra i protagonisti Dialta, da tre anni vedova di Domenico de Mayda. Vivo il marito di Dialta, Stefano de Lepira de Apriliano di Crotone appellabat in commatrem la donna; morto Domenico, Stefano aveva preso Dialta in concubinam et cum ea procreavit unam filiam. Saputo che il cognato aveva accusato Stefano di omicidio, Dialta esprimeva puplice il suo pensiero: quisto bastardo auchise mio marito, et promisemi di farimi honori et mo mi ha gabato. Alle accuse seguiva un allontanamento fuori Crotone della donna, raggiunta a breve da Stefano, con prevedibili conseguenze: Dialta veniva trovata morta ipso denunciato existente cum eadem ${ }^{31}$.

Nel letto mentre dormiva veniva strangolata et nequiter interfecta Giovanna, trovata morta l'indomani: colpevole il marito, Pressano figlio de lo Tosco de Castrofranco, che fuggiva portando con sé quamplura bona della donna $^{32}$.

Di Cosenza era Pietro Czurella denunciato da Impernata, vedova di Giovanni Carmeta che più volte, preconiczando et vociferando ad iniuram et pro iniuria della donna aveva detto: hagio dormito in meczo de la matria et de la figlastra, e mala malis agregando aveva chiamato Impernata: previtera e bastarda. Ingiurie che miravano a colpire la provenienza sociale ma anche - previtera - a bollare la donna macchiatasi di frequentazioni intime con i religiosi $^{33}$. Nei confronti di Pietro Impernata aveva sporto ulteriore denuncia, preoccupata del fatto che quello girava armato, ascia alla mano, animo et intencione di aggredirla; e un'altra denuncia era partita dalla figlia di Impernata, Caterinella cui Pietro aveva detto chi Andria soy figlo la havia canosuta carnaliter ${ }^{34}$.

Devianze, quelle a carattere sessuale, pericolose per il mantenimento dell'ordine sociale: il rischio dell'adulterio, e del concubinato - decine di casi, tra le carte del nostro registro- era infatti la perdita dell'onorabilità del matrimonio, con ricadute sul contesto familiare, e non solo. Tommaso

\footnotetext{
${ }^{29}$ ASN, Dipendenza, vol. 631, c. 9.

${ }^{30}$ ASN, Dipendenza, vol. 631, c. 66.

${ }^{31}$ ASN, Dipendenza, vol. 631 , c. 35 .

${ }^{32}$ ASN, Dipendenza, vol. 631, c. 51.

${ }^{33}$ Riscontrata in parecchie aree, dal Piemonte alla Toscana alla Sicilia, anche l'ingiuria " $f i l i u$ di previti", figlio di prete (nella versione al femminile: "preveressa" "pretessa"), con riferimento alle intemperanze sessuali dei religiosi; cfr. Acta Curie Felicis Urbis Panormi, 5, Registri di lettere ed atti (1328-1333), a c. di Pietro CORRAO, Palermo, 1986, doc. 5, p. 14; NADA PATRONE, Simbologia e realtà, p. 51. V. inoltre Salvatore BONGI, Ingiurie, improperi, contumelie: saggio di lingua parlata del Trecento cavato dai libri criminali di Lucca, Bologna, 1890 , n. ed. Lucca, 1983.

${ }^{34}$ ASN, Dipendenza, vol. 631, c. 39.
} 
Sellario, napoletano, denunciava Giovanni Maccarono di Cosenza macchiatosi di adulterio con Tucia, moglie di Colella e famula del viceré; un adulterio che veniva commesso in casa di Tommaso, absque consciencia et voluntate dello stesso. In quell'occasione Tommaso denunciava anche Pacilia, moglie del magister Nicola de Patrino macchiatasi con il marito di lenocinio in domo habitacionis ipsius Thomasii ${ }^{35}$. Colpevole di adulterio Crissio de Desio, di Pedace: contratto matrimonio in Albania con Caterina e consumate le nozze, tornato in patria sposava animo leto l'ignara Brigida ${ }^{36}$. Flora era invece stata ingannata da Antonio Ammucharicio di San Pietro che l'aveva persuasa a una conoscenza carnale con la promessa di sposarla, aveva con lei procreato una figlia e poi aveva preso in moglie un'altra donna, lasciando la denunciatrice inanem et vacuam ${ }^{37}$.

Il capitano di San Mauro, Bernardo de Casello de Sancto Marco, veniva denunciato alla curia da Giacomo Antonio de Zaffiro, di Rossano: aveva fatto rapire la figlia illegittima di questi, Maffia, e condurre a San Mauro tenendola in suam amasiam nomine meretricis ${ }^{38}$.

Una violenza, non di rado, tra donne. Caterina, moglie di Giovanni Grande di Rogliano nel marzo 1454 veniva denunciata per avere assalito di notte Fronda mentre questa si trovava in casa: nel tentativo di soffocarla, la accecava ${ }^{39}$. Violante, moglie di Stefano Battista di Cosenza aveva aggredito Rosa Castrovillari che pacifice et quiete neminem iniuriam inferendo si era trovata a passare davanti casa sua e l'aveva colpita con una pietra in testa cum tumore et vulneris apparicione: in un'escaletion di violenza Violante volens tunc ibidem sue pravissime et inique voluntate satisfacere, afferrata Rosa per la mano sinistra, l'aveva morsa a sangue e se non fossero accorsi prontamente, l'avrebbe colpita a morte. La donna era quindi passata agli oltraggi e, animo iniurandi et eidem iniuriam inferendi, aveva chiamato Rosa puctana ruffiana ${ }^{40}$. A Cosenza Isolda era stata denunciata da Giuliano Fabario per le parole -ingiuriose anche nei suoi confronti- rivolte alla moglie Primarosa: poctana sculenterata conventata chi non te hassata franczosi nè catalani ${ }^{41}$.

Tra meretrici, frequenti sembrerebbero le accuse di bestemmia: veniva denunciata alla curia del viceré Angela meretrix che blasfemavit Deum un venerdì di novembre; Guerchia veniva accusata da Ventura, entrambe prostitute, perché blasfemavit Deum davanti la bottega Florismontis, a

\footnotetext{
${ }^{35}$ ASN, Dipendenza, vol. 631, cc. 30-31.

${ }^{36}$ ASN, Dipendenza, vol. 631, c. 6.

${ }^{37}$ ASN, Dipendenza, vol. 631 , c. 45 .

${ }^{38}$ ASN, Dipendenza, vol. 631 , c. 51.

${ }^{39}$ ASN, Dipendenza, vol. 631 , c. 42.

${ }^{40}$ ASN, Dipendenza, vol. 631 , c. 30

${ }^{41}$ ASN, Dipendenza, vol. 631 , c. 89.
} 
Cosenza $^{42}$. Ancora a Cosenza, Filippa denunciava una concittadina che, accusata di avere ucciso una sua gallina, aveva bestemmiato San Giacomo ${ }^{43}$.

\section{VitA IN CALABRIA NEL XV SECOLO: UNO SPACCATO SOCIALE}

Un clima di aggressività si respira nelle città del XV secolo, in cui la violenza diventa parte di un ordine naturale delle $\operatorname{cose}^{44}$. Una società, parrebbe, ovunque sottomessa e obbediente «in linea verticale» ma «assai litigiosa in linea orizzontale ${ }^{45}$. Vita particolarmente violenta in Calabria, regione che «nell'estremità meridionale del Regno di Napoli e quindi della penisola italica, subiva, per effetto di tale posizione geografica, anche il peso d'un certo isolamento dai centri più progrediti o in via di progresso»: tra cui, i due 'varchi' privilegiati, Napoli e Messina ${ }^{46}$.

Rapporti dunque improntati alla vendetta, alla ritorsione, alla faida perfino, specie quando l'offesa arrecata a un singolo è diretta alla sua famiglia, con la conseguenza di generare una vera e propria guerra privata tra gli schieramenti ${ }^{47}$. Il principe Galgano de Rogliano nel marzo 1454, a Cosenza, era stato aggredito cum quadam runcha da Antonio de Sancto Petro, animo et proposito ipsum acriter percutiendi: il principe teneva in concubinam una donna di nome Tomasia, moglie dello scomparso Nicola Pecuri e commatrem dell'assalitore ${ }^{48}$.

Dietro denunce e aggressioni si celano moventi o sfumature interiori in apparenza insondabili e, la maggior parte delle volte, retroscena sconosciuti: cosa, per esempio, si celava dietro l'uccisione dei figli (due maschi e una femmina) di Agnese, Giovanna, Flora, tre donne di Squillace che denunciavano la cosa al vicerée ${ }^{49}$. Angelo Cussino, mentre si trovava in prossimità della chiesa dei Santi Filippo e Giacomo, a Cosenza, subiva l'aggressione di Francesco Bove e del figlio di questi, Pietro: i due lo avevano afferrato per capillos ipsumque acriter verberaverunt in facie, capite et corpore et eciam percusserunt eum in tibia cum lapidibus cum livore, tumore et sanguinis effusion $e^{50}$. Più che affidarsi alla giustizia, l'individuo che si ritiene offeso, schernito, vessato, ricorre alla violenza: una furia dell'animo che si traduce

${ }^{42}$ ASN, Dipendenza, vol. 631, cc. $11,64$.

${ }^{43}$ ASN, Dipendenza, vol. 631, c. 88.

${ }^{44}$ Lauro MARTINES, Violence and Civil disorder in Italian Cities 1200-1500, UCLA, Los Angeles, 1972, pp. 3-7'e 310-330.

${ }^{45}$ NADA PATRONE, Simbologia e realtà, p. 55.

${ }^{46}$ PONTIERI, La Calabria, p. 61. Sulla violenza della vita messinese, Daniela SANTORO, Messina l'indomita. Strategie familiari del patriziato urbano tra XIV e XV secolo, CaltanissettaRoma, 2003, pp. 59 ss.

${ }^{47}$ Cfr. Jacques HeERS, Il clan familiare nel Medioevo, Napoli, 1976, pp. 150 ss.

${ }^{48}$ ASN, Dipendenza, vol. 631, c. 40.

${ }^{49}$ ASN, Dipendenza, vol. 631, c. 37.

${ }^{50} \mathrm{ASN}$, Dipendenza, vol. 631, c. 30 . 
in tentativi di omicidio spesso riusciti e che innesca situazioni di scontro tra le parti tali da rendere estremamente rara la possibilità di una via di soluzione.

Motivi economici, di difesa dell'onore familiare, bramosia di denaro, invidia, diffamazioni, piccole vendette e antipatie, dispetti, ripicche tra persone legate da rapporti di lavoro, di vicinato: io non voglo ruffiana nè puctana nè missagera ananczo la porta di la casa mia aveva detto Pietro Franczoso a Rosa de Agustino che abitava a Cosenza, vicino la casa del notaio Simone de Docimo ${ }^{51}$. Varie, e al contempo fissate e sospese nello spazio e nel tempo, sembrano essere le cause che fanno scattare una denuncia che difficilmente si risolve con una composizione tra le parti. Sospettato di furto, Paolo de Olivero aveva denunciato Nicola Ipolito di Serra Pedace che animo iniurandi lo aveva accusato: Paulo tu ay piglata la gallina mia cum lo bastoni alla vigna mia, credi di tila mangiari como te mangiasti la pecura di notari Petri di Sillitta de Serra Pedaci?, aggiungendo ulteriori offese. Un'altra denuncia era partita lo stesso giorno nei confronti del conterraneo Giovanni de Lucia poichè, ad iniuriam, conthumeliam et iacturam dello stesso, aveva detto chi Paulo me have raputo uno porcello de li mey ${ }^{52}$. Impegnato a allontanare la sporcizia accumulatasi davanti casa, Apostolo Cocio era stato assalito e e colpito, sul costato con una pietra, e poi sul capo e nel viso, a sangue, da Giovanni e Pressano Lupinaro, di Paterno ${ }^{53}$.

Intenta alla raccolta delle castagne - la metà che le spettava secondo quanto stabilito- in Castello Gelani, vicino Pedace, Caterina veniva aggredita da Consulo Celistina di Celico che le spaccava la testa con un bastone ${ }^{54}$. Alberi di castagno - «principale supporto dell'orizzonte calabrese», la diffusione era massiccia e distribuita in modo uniforme sul territorio calabrese a fornire legno e cibo per uomini e animali ${ }^{55}$ - fanno da sfondo alla denuncia che il notaio Pirosino de Nicolecta di Santo Stefano sporgeva nei confronti del cosentino Bernardo Mancino, accusato di avere tagliato e rubato legna da un albero castanearum fructiferam che si trovava all'interno di una vigna di proprietà del notaio ${ }^{56}$.

Aggressori e vittime anche alcuni membri della comunità ebraica. Nel marzo 1454, Zacha Fossana, ebrea di Cosenza, aveva denunciato un concittadino ebreo che l'aveva assalita mentre si trovava in via puplica seu strata iudeorum, ossia nella giudecca di Cosenza, afferrandola per la gola modo ipsam suffocandi, ponendo pollices in gucturem et razando seu rigando a sangue la donna: a deporre venivano chiamati come testimoni altri membri della comunità ${ }^{57}$.

${ }^{51}$ ASN, Dipendenza, vol. 631, c. 81.

${ }^{52}$ ASN, Dipendenza, vol. 631, cc. 27, 28.

${ }^{53}$ ASN, Dipendenza, vol. 631, c. 32.

${ }^{54} \mathrm{ASN}$, Dipendenza, vol. 631, c. 11.

${ }^{55}$ Carmela Maria Rugolo, Paesaggio boschivo e insediamenti umani nella Calabria medievale, in «Il bosco nel medioevo», a c. di Andreolli-Montanari, Bologna, 1995, p. 264.

${ }^{56}$ ASN, Dipendenza, vol. 631, c. 46

${ }^{57}$ ASN, Dipendenza, vol. 631, c. 41. Cfr. Colesanti, Documenti, pp. 29 ss. 
Gesti, a volte, non eccessivamente violenti ma carichi di simboli, e offensivi, come gli schiaffi dati pubblicamente: cosa che aveva fatto scattare la denuncia per il giudice Giacomo di Amantea, dal famulo di Antonio Caputo $^{58}$. In un caso, poi, abbiamo la possibilità di visualizzare la reazione alla pena: denunciato in seguito a una 'sottrazione' di buoi utilizzati per arare un terreno senza il permesso del proprietario, Giacomo Romero di Crotone era raggiunto dall'ordine di recarsi davanti a viceré e capitano di Crotone. A segnale inequivocabile del suo pensiero, raggiunto dal mandato di comparizione Giacomo aveva reagito: quista pena la tineo quanto quista, buttando - il gesto più forte, e talora sprezzante, della parola-dell'immondizia per terra ${ }^{59}$.

Una violenta quotidianità in cui alle violenze fisiche - con pugni e calci, a mano nuda o con bastoni, asce, scuri, balestre, lame, zappe, ronconi, coltelli, pietre, persino con un pezzo di carne come era successo nel luglio 1454 a Matteo de la Sellia o con una pietra di sale ${ }^{60}$-si sposano quelle verbali, con una terminologia che «è lo specchio della risposta simbolica di una società di fronte al suo vissuto quotidiano». Offese che, ai fini di una ricostruzione à part entière, offrono «la possibilità di illuminare le scelte di comportamento e le reazioni psicologiche di un gruppo sociale» ${ }^{61}$. Ampio appare, nelle carte del nostro registro, il ventaglio di insulti: ravagloso de merda, dementi bastarda, mala cane; i più scontati e frequenti puctana, ruffiana attinenti alla sfera sessuale ${ }^{62}$; e poi gli insulti riguardanti il comportamento sociale, latro soprattutto -epiteto con il quale Francesco Sicario di Cosenza si era rivolto a Nicola Montalto, ed era stato per questo denunciato $^{63}$ - ma anche scassature de casa, insulto (da non escludere un senso figurato), che si era sentito rivolgere un prete ${ }^{64}$. Sporgeva denuncia nei confronti di donna Prima Rosa, Isolda, moglie di Francesco Bono di Cosenza: mentre si trovava in un casale in prossimità di Cosenza et ibidem faceret ministerium sete seu laboraret setam pacifice et quiete, Isolda era stata assalita da quella che gerens malivolum animum nei suoi confronti, recatasi al casale con un bastone seu fusto lanczee aveva distrutto e raso al suolo logiam sistentem in dicto casaleno e le aveva indirizzato parole ingiuriose: gagloffa femmena demente inbriaca ${ }^{65}$.

Studio, quello delle violenze verbali, che non solo consente di indagare «la struttura specifica della mentalità e della psicologia individuale e collettiva e della simbologia delle parole» ma che può costituire un «utile punto di riferimento per cogliere l'atteggiamento mentale che faceva

${ }^{58}$ ASN, Dipendenza, vol. 631, c. 9.

${ }^{59}$ ASN, Dipendenza, vol. 631, cc. 33-34.

${ }^{60} \mathrm{ASN}$, Dipendenza, vol. 631, cc. $73,84$.

${ }^{61}$ NADA PATRONE, Simbologia e realtà, p. 57.

${ }^{62}$ ASN, Dipendenza, vol. 631, cc. 8, 9, 30, 81. V. Gerhard RoHLFs, Dizionario dialettale delle tre Calabrie, Milano, 1932.

${ }^{63}$ ASN, Dipendenza, vol. 631, c. 40.

${ }^{64}$ ASN, Dipendenza, vol. 631, c. 65 .

${ }^{65}$ ASN, Dipendenza, vol. 631, c. 88. 
considerare reati maggiormente gravi certi epiteti e quindi meritevoli di multe più pesanti o di pene di mortificazione o di punizioni fisiche»: per giungere alla conclusione, sono parole di Nada Patrone, dell'esistenza di 'modelli' di ingiurie «validi in tutta l'Italia tardo-medievale» ${ }^{66}$.

Le offese verbali sono spesso accompagnate dalle minacce: eo ti farò dare cento bastonate, aveva detto Sirisi, di Crotone, a Pietro Labruto, aggredendolo mentre questi in prossimità della porta della città ubi dicitur burgo stava portando il suo cavallo ad abbeverarsi, e aveva pronunciato parole ingiuriose - bructo cornuto tradditore eo te sgallero uno corno-, aggiungendo ad suam maximam iniuriam et vilipendium un'ulteriore minaccia: io ti taglerò lo naso de la fache ${ }^{67}$. Francesco Martorano de Turri Insule era stato denunciato da frate Giovanni de Ariano monaco del monastero di Santa Maria de Corachio e procuratore dell'abate: mentre si trovava in un terreno in prossimità terre Castellorum, impegnato a portare avanti, come era solito, facta et negocia, dell'abate e del monastero, dividendo et liberando erbas del terreno, veniva aggredito da Francesco armato con una balestra parata cum quadam latula; intenzionato ad impedirgli di svolgere il suo compito, si rivolgeva minaccioso al frate e agli astanti: per la fide mia tucti ve auchido ${ }^{68}$. Offuscamenti e alterazioni che si traducono in minacce, o promesse, di morte: io te chuderò quista buccacza cha non parlerai tanto aveva detto ampullosus Angelo de Santo Blasio al cosentino Giovanni Amantea, annunciando avanti chi sia octo dy io te troverò et chuderoti quissa bucca ${ }^{69}$. Nicola Guerrerio, di Cosenza, nel gennaio 1454 aveva assalito cum pugillo in oculo Angelo Feczano mentre si trovava in una bottega: colpevole, Angelo, di avere pronunciato parole in difesa di donna Elisabetta che aveva litigato con la moglie dell'assalitore; un oltraggio che Nicola non aveva sopportato e indirizzate nei confronti di Angelo parole ingiuriose - succzo mulo arribaldo mi veni vogla de ti caczari tucti li denti- lo aveva afferrato per i capelli ripetendo ossessivamente la sua minaccia ${ }^{70}$.

Naso e denti sono la parte fisica su cui più frequente si concentra l'attenzione degli accusati, ma non mancano intenzioni originali: io te allargerò la pelle aveva detto Nicola de Corrado di Motta, denunciato da Roberto servitore dell'abbazia di San Giovanni de Paliaris che abitava a Celico $^{71}$.

Una criminalità, nel complesso, povera di motivazioni politiche, espressione di una parte di società cui erano precluse ingerenze e presenza nella vita politica. Terra, la Calabria, destinata a rimanere quasi 'zona franca',

\footnotetext{
${ }^{66}$ NAdA Patrone, Simbologia e realtà, pp. 47 s., per un esemplio di ibidem, pp. 71 ss. le tabelle con le varie tipologia di ingiurie e Giorgetta BONFIGLIO DOSIO, Criminalità ed emarginazione a Brescia nel primo Quattrocento, Firenze, 1978, p. 115.

${ }^{67}$ ASN, Dipendenza, vol. 631, c. 23.

${ }^{68}$ ASN, Dipendenza, vol. 631, c. 33.

${ }^{69}$ ASN, Dipendenza, vol. 631, c. 55.

${ }^{70}$ ASN, Dipendenza, vol. 631, cc. 27-28.

${ }^{71}$ ASN, Dipendenza, vol. 631, c. 73.
} 
data la densità della tendenza a delinquere che in verità, non era prerogativa dei soli calabresi: abitava a Cosenza il catalano Giovanni de Fundi et Cardona, denunciato da Guglielmo Raimondo de Flumere de regno Valencie. Giovanni aveva giocato cum taxellis ad aczardum con Guglielmo Raimondo a Cosenza, nel fondaco di San Benedetto e aveva vinto quattro ducati e mezzo. Il valenzano scopriva però di essere stato truffato dal momento che, astuto, il catalano aveva portato con sé taxillos falsos seu de avantagio ${ }^{72}$.

Con fatica, in una Calabria isolata e distante, poteva mettere radici e trovare realizzazione il luminoso ideale di giustizia previsto dal programma di Alfonso V che nei confronti dei calabresi, si ricordava, avrebbe nutrito sentimenti ostili, ricambiati parrebbe: Gabriele di Sanseverino di Cosenza era stato denunciato da Antonio Ventura, servitore della curia, per avere replicato, a quello che minacciato si era appellato alla maestà del re e del viceré: io non hagio pensero nè de lo re nè de lo vicerré, bructo latro arribaldo gagloffo ${ }^{73}$. Nel maggio 1454 -erano trascorsi dodici anni dal trionfale ingresso di Alfonso a Napoli- Giacomo Benincasa di Mangone veniva denunciato da Giovanni Caczoferrato: lo aveva aggredito cum armis prohibitis e tentato, la moglie di Giovanni sulla soglia, di entrare con violenza in casa sua. Un'altra accusa pendeva su Giacomo denunciato nuovamente da Giovanni che si era sentito dire non ti lasso venire alla casa ca mi facivi dire 'viva Re $d d^{\circ}$ Aragona' ad forcza $a^{74}$ : sonora conferma di quella reciproca idiosincrasia che correva tra Alfonso e i calabresi.

Data di ricezione dell'articolo: maggio 2008.

Data di accettazione e versione finale: luglio 2008.

\footnotetext{
${ }^{72}$ ASN, Dipendenza, vol. 631, c. 12. Sul gioco d'azzardo v. Ludovico ZDEKAUER, Il gioco d'azzardo nel Medioevo italiano, con saggio introduttivo di Gherardo ORTALLI Firenze, 1993 Giovanni CECCARELLI, Il gioco e il peccato. Economia e rischio nel Tardo Medioevo, Bologna, 2003.

${ }^{73}$ ASN, Dipendenza, vol. 631 , c. 83.

${ }^{74}$ ASN, Dipendenza, vol. 631 , c. 55 .
} 